\title{
Viscous micropump of immiscible fluids using magnetohydrodynamic effects and a power-law conducting fluid
}

\author{
J. Gómez ${ }^{a}$, C. Hernández ${ }^{b}$, J. Escandón ${ }^{a, *}$ and R.O. Vargas ${ }^{a}$ \\ ${ }^{a}$ Instituto Politécnico Nacional, SEPI-ESIME Azcapotzalco, Departamento de Termofluidos, \\ Av. de las Granjas No. 682, Col. Santa Catarina, Alcaldía Azcapotzalco, Ciudad de México, 02250, Mexico. \\ *e-mail: jescandon@ipn.mx \\ ${ }^{b}$ Universidad Tecnológica de México, UNITEC MÉXICO-Campus Marina-Cuitláhuac, \\ Ciudad de México, 02870, Mexico
}

Received 5 June 2021; accepted 2 July 2021

\begin{abstract}
Small-scale fluid transport methods have grown significantly in recent years, mainly in microfluidic system applications. Therefore, the present study analyzes the movement of two layers of immiscible fluids within a parallel flat plate microchannel. The fluid layers are composed of a Newtonian fluid and a power-law fluid. The pumping is produced by magnetohydrodynamic effects that act on the non-Newtonian conducting fluid dragging the non-conducting Newtonian fluid by viscous forces. Under the consideration of a laminar, incompressible, and unidirectional flow, a dimensionless mathematical model is established by the momentum equations for each fluid, together with the corresponding boundary conditions at solid-liquid and liquid-liquid interfaces. The problem formulation is semi-analytically solved using the Newton-Raphson method. The results are presented as a function of the velocity profiles and flow rate, showing interesting behaviors that depend on the physical and electrical properties of each fluid and flow conditions via the dimensionless parameters such as the flow behavior index, a magnetic parameter related to Lorentz forces, the fluid viscosity ratios and the dimensionless liquid-liquid interface position. This work contributes to the understanding of the various immiscible non-conducting fluid pumping techniques that can be used in microdevices.
\end{abstract}

Keywords: Micropump; non-Newtonian fluid; immiscible fluids; magnetohydrodynamics; parallel flat plates microchannel.

PACS: 47.55.-t; 47.85.-g; 68.05.-n; 68.08.-p; 41.20.-q

DOI: https://doi.org/10.31349/RevMexFis.67.060601

\section{Introduction}

Magnetohydrodynamics (MHD) pertains to flows of electrically conducting fluids that are subject to a magnetic field and/or an electric current driven by an external voltage [1]. Since the last century, MHD has been used in various scientific and technological applications such as heating, stirring and levitating liquid metals, magnetic damping, flow control by magnetic throttles, and electromagnetic flow meters. Other applications are related to MHD generators and pumps, continuous casting, liquid metal shaping, and design and construction of liquid metal cooled fusion blankets in nuclear fusion reactors, among others [1,2]. Typical fluids of MHD studies are distilled water $\left(10^{-4}\right)$, weak $\left(10^{-4}-10^{-2}\right)$ and strong $\left(10^{-2}-10^{2}\right)$ electrolytes, molten glass $\left(10-10^{2}\right)$, plasmas $\left(10^{3}-10^{6}\right)$, ionized gases $\left(10^{7}\right)$, and liquid metals $\left(10^{6}-10^{7}\right)$. The numbers in parentheses refer to the electrical conductivity of the materials in $\mathrm{S} \mathrm{m}^{-1}$ [3].

Focusing on MHD pumping, this method of transporting material offers some advantages and disadvantages over conventional pumps. The benefits of MHD pumps are that they are simple and compact, as well as silent due to propulsion without any moving parts [4]. They have simple fabrication processes, lower actuation voltages, reduced risk of clogging and damage to molecular materials, reduced risks of mechanical fatigue, and continuous fluid flow [5]. Additionally, they can withstand very high-temperature environ- ments as in the cases of molten metals $(\mathrm{Na}, \mathrm{Pb})$ and their alloys $(\mathrm{Pb}-\mathrm{Bi})$ or molten salts (especially fluoride salts) $[6,7]$. On the other hand, they present certain disadvantages such as reverse flow due to the fringing fields effects, the major expense of large magnets, a lack of accurate analytical models [4], a short electrode lifetime and bubble generation due to electrolysis [8]. The advantages and disadvantages of MHD pumps mentioned above may be related to microscale and macroscale applications. However, it is necessary to clarify that the present work is focused only on microdevices.

In this context, with the technological advancements in the miniaturization of the devices (bioMEMS - biological microelectromechanical systems, $\mu$ TAS - micro total analysis systems, LOCs - labs on chips) for analysis and diagnostics in the chemical, medical and biological areas, the study of fluid flow has become essential. Here, research on submillimeter-dimension devices is covered by microfluidics together with the application of magnetohydrodynamics as a method of transporting conducting fluids [9-13].

Considering the previous application areas, the study of MHD flows on the microscale has been addressed by the scientific community for many years, through investigations on transport of homogeneous single-phase fluids based on electrolyte solutions. Some of these investigations are described below. Lim and Choi [14] realize an analytical, numerical, and experimental study about an MHD micropump formed by a trapezoidal microchannel where the working fluid is a 
PBS solution (phosphate-buffered saline). In this study, the performance of the MHD micropump is obtained by measuring the flow rate under various operation currents and magnetic flux densities. For their part, Huang et al. [15] report a theoretical and experimental work on a DC-type MHD micropump using working fluids such as sodium hydroxide $(\mathrm{NaOH})$ in water, ammonium chloride $\left(\mathrm{NH}_{4} \mathrm{Cl}\right)$ in water, ethyl alcohol, and tap water. Here, the average flow rates measured for a range of applied potentials are affected by the generation of bubbles due to electrolysis. In another investigation, Patel and Kassegne [5] conduct an analytical effort to investigate different flow channel geometries, effects of nonuniform magnetic and electric fields, Joule heating, and electroosmosis in MHD micropumps; here, they use saline solutions based on sodium chloride $(\mathrm{NaCl})$. The reader can review other investigations on MHD micropumps handling homogeneous single-phase fluids such as the studies conducted by Homsy et al. [11], Chatterjee and Amiroudine [16], Bau et al. [17], Rivero [18], Azimi-Boulali et al. [19], Moghaddam [20], Jian and Chang [21], Mondal and Wongwises [22], and many others cited in these works.

A common characteristic of all the investigations mentioned in the previous paragraph is that they all use fluids with Newtonian behavior. However, several biological or polymeric fluids present a non-Newtonian behavior, which has also been investigated for MHD pumps, as in the study performed by Moghaddam [23], who models the transport of power-law fluids. The work reports that the flow behavior index has a strong effect on the volumetric flow rate in the MHD micropump. Pourjafar et al. [24] conduct a numerical investigation of an MHD micropump wherein the test fluid is assumed to be viscoplastic obeying the PapanastasiouBingham rheological model. Their results suggest that a the yield stress of a fluid can dramatically affect the flow kinematics in MHD pumps, even under creep conditions. Additionally, it is predicted that the M-shaped velocity profiles established in the literature for Newtonian fluids become virtually U-shaped, provided that the Bingham number is sufficiently large. Other studies about the importance of considering the non-Newtonian behavior of fluids in MHD micropumps are discussed in references [25-27]. In the aforementioned investigations, different values of the magnetic, material, and physical parameters, in conjunction with complex rheological properties of the fluids, help control the flow field.

In this direction, the research on MHD flows with singlephase fluids has also been extended to the pumping of two parallel and immiscible fluids as a transport technique by viscous drag or for improvement of the flow rate of the pumping fluids. For this type of research, only the driving fluid is electrically conducting. The study of Shail [28] considers the Hartmann flow of a conducting fluid together with a layer of non-conducting fluid in a channel formed by two parallel plates. The interest in this configuration stems from the possibility of reducing the power required to pump oil in a pipeline through the addition of water. According to the results, the flow rate can increase by approximately 30 percent for suitable ratios of the thicknesses and viscosities of the two fluids. In other work, Lohrasbi and Sahai [29] study the two-phase MHD flow and heat transfer in a parallel-plate channel to understand the effects of slag layers on the heat transfer characteristics of a coil-fired MHD generator. In the problem, one of the two fluids is electrically non-conducting, and the flow is incompressible, steady, one-dimensional, and fully developed. In the results, velocity profiles as a function of dimensionless parameters (magnetic, electrical, and physical) are presented. Umavathi et al. [30] extend the investigations of Shail [28] and Lohrasbi and Sahai [29] to solve the unsteady Hartmann flow of two immiscible fluids through a horizontal channel. This study finds that the properties of the two fluids can effectively control the flow and heat transfer characteristics. In another work and to improve the fluid flow in pipelines, Mateen [31] develops a theoretical analysis of an MHD flow of two immiscible fluids taking into account the effects of viscous dissipation and Joule heating. In this sense, as a method for enhancement of heat transfer, Abbas and Hasnain [32] study the two-phase magnetoconvection flow of nanoparticles in a horizontal composite annulus. Here, the electrically conducting fluid is a kerosene-based ferrofluid containing ferroparticles of magnetite $\left(\mathrm{Fe}_{3} \mathrm{O}_{4}\right)$. The velocity and temperature distributions under the influence of involved flow parameters and volume fraction of nanoparticles are analyzed and discussed. In other investigations, some applications of two-layered MHD flows in vertical and inclined channels such as heat transfer analysis, solar collection technology, and radiative cooling of molten glass are analyzed by Abbas et al. [33], Malashetty and Umavathi [34], and Malashetty et al. [35]. Keeping in view the practical importance of multi-fluid flows in chemical, nuclear and petroleum industries, and in magnetofluid dynamics, Nikodijevic et al. [36] investigate the MHD Couette flow and heat transfer of two immiscible fluids in a parallel-plate channel. Closedform solutions for velocity, temperature, and magnetic induction are obtained in the presence of applied electric and inclined magnetic fields. In another application, Chanturani and Bharatiya [37] investigate the two-layered MHD model for parallel-plate hemodialyzers under the influence of a uniform transverse magnetic field. The results can be used in reducing the dialysis time. Here, the transported blood consists of a suspension of red cells and plasma. This suspension is divided into two Newtonian fluid layers. The first is the peripheral plasma layer as non-conducting fluid, and the second layer is the core region consisting of cells and plasma as the conducting fluid. The applications mentioned throughout this paragraph regarding two-layer MHD flows have been focused on the macroscale and handling only Newtonian fluids. Therefore, for the reader's better knowledge, an extensive review on applying two-layer MHD flows has been carried out in this investigation. It has been concluded that little or no attention has been paid to this flow type on the microscale with respect to pumping non-conducting fluids by viscous drag.

Despite the above, viscous drag pumping in microfluidic 
devices is being addressed by electrokinetics. Some investigations about electrokinetic flows of two layers consider the transport of Newtonian [38-40] and non-Newtonian fluids [41-43]; however, this is limited for weak electrolytes $\left(<0.1 \mathrm{~S} \mathrm{~m}^{-1}\right)$. Thus, the pumping by viscous drag effects caused by MHD flows for the transport of weak and strong electrolytes $\left(>0.1 \mathrm{~S} \mathrm{~m}^{-1}\right)$ in microdevices is currently an open and unexplored field.

Therefore, the aim of this work is focused on analyzing a DC MHD micropump that transports a non-conducting fluid via viscous drag forces from a neighboring conducting fluid in a microchannel. Additionally, the conducting fluid presents characteristics of a power-law fluid, which can be used to explore the influence of the fluid's rheology in improving the pumping flow rate. The parametric study presented here is an investigation that has not been carried out yet by the scientific community.

\section{Problem formulation}

\subsection{Physical model}

The present work analyzes the transport of two immiscible fluids in a parallel flat plate microchannel of height $H$, width $W$ and length $L$, as shown in Fig. 1. The Cartesian coordinate system $(x, y, z)$ is located at the bottom of the channel inlet. Fluid pumping primarily occurs through MHD effects; however, a pressure differential can be generated between the inlet and outlet of the microchannel $\left(\Delta p=p_{\text {out }}-p_{\text {in }}\right)$, producing a common constant pressure gradient over fluid layers. The flow field is made up of two layers of fluids divided by the interface position $y_{1}$. For the range of $0<y<y_{1}$, there is a Newtonian fluid (driven fluid), while for the range of $y_{1}<y<H$, there is a non-Newtonian fluid (driving fluid) composed of a mixture of an ionized solution and a solute which behaves like a power-law fluid. Here, $B_{y}$ on the $y$-axis is the magnetic field from permanent magnets located at the upper and bottom walls of the microchannel, and $J_{z}$ on

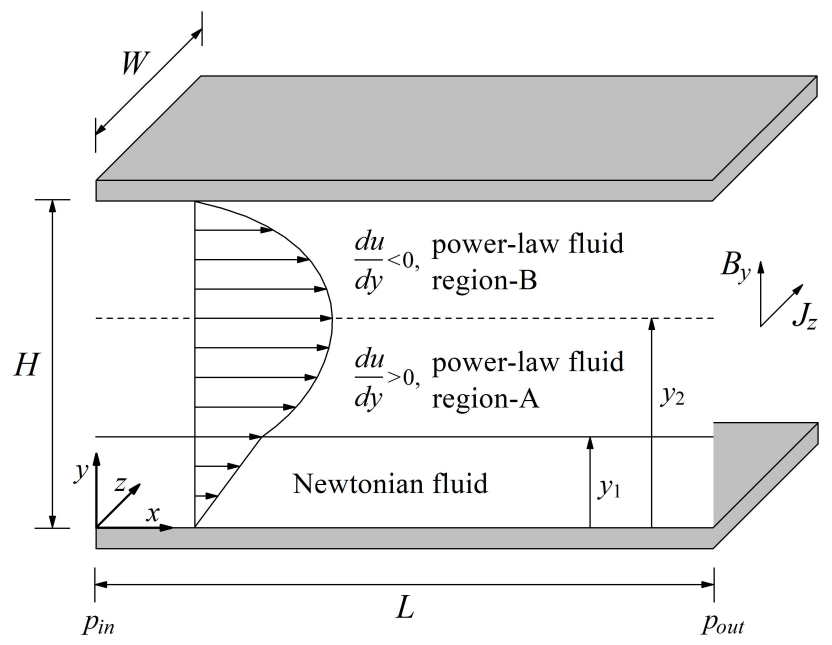

FIGURE 1. Sketch of the magnetohydrodynamic flow in a parallel flat plate microchannel. the $z$-axis is the electric current density generated by an electric field from a pair of electrodes placed at the lateral walls; these interact transversally to each other to produce the Lorentz forces that act on the electrically conducting nonNewtonian fluid, resulting in dragging of the electrically nonconducting Newtonian fluid by viscous forces. On the other hand, the power-law fluid layer can be divided into two regions $\mathrm{A}$ and $\mathrm{B}$, showing the velocity gradient behavior regarding the increase $(d u / d y>0)$ and decrease $(d u / d y<0)$ of the velocity profile slope. The distance $y_{2}$ as a fictitious interface indicates the point at which the maximum velocity of the non-Newtonian fluid is reached.

\subsection{General governing equations}

The governing equations for incompressible fluids that describe the flow field are given by the continuity equation

$$
\nabla \cdot \mathbf{v}=0
$$

and the Cauchy momentum equation

$$
\rho \frac{D \mathbf{v}}{D t}=-\nabla p-\nabla \cdot \boldsymbol{\tau}+\mathbf{F},
$$

where $\mathbf{v}$ is the velocity vector, $\rho$ is the fluid density, $t$ is the time, $p$ is the pressure, $\boldsymbol{\tau}$ is the stress tensor, and $\mathbf{F}$ is the body force vector. Here, body forces appear due to the application of the electric and magnetic fields; their magnitude is determined by

$$
\mathbf{F}=\mathbf{J} \times \mathbf{B}
$$

where $\mathbf{J}$ represents the electric current density given by Ohm's law as $\mathbf{J}=\sigma(\mathbf{E}+\mathbf{V} \times \mathbf{B}) ; \sigma$ denotes the electric conductivity, and $\mathbf{E}$ and $\mathbf{B}$ are the electric and magnetic field vectors, respectively. On the other hand, the generalized constitutive equation for the power-law fluid is expressed as

$$
\tau=-\eta(\dot{\gamma}) \dot{\gamma}
$$

where $\eta$ is the dynamic viscosity and $\dot{\gamma}$ is the rate-of-strain tensor defined as $\dot{\gamma}=\nabla \mathbf{v}+(\nabla \mathbf{v})^{\mathbf{T}}$. Furthermore, the viscosity for fluids that follow a behavior based on the rheological power-law model is

$$
\eta(\dot{\gamma})=m \dot{\gamma}^{n-1},
$$

where $m$ is the flow consistency coefficient and $n$ is the flow behavior index. In the case of a Newtonian fluid with constant viscosity $\mu$, from Eq. (4), $\eta(\dot{\gamma})=\mu$.

\subsection{Simplified mathematical model}

The mathematical model used to solve the MHD flow can be simplified by taking into account the following considerations: $i$ ) Steady, laminar and fully developed flow is assumed $[5,12,14,34]$. ii) The electrical and physical properties of fluids are assumed to be constant [32,44,45]. iii) Laminar flow is assumed for low Reynolds number, i.e., 
$\operatorname{Re}\left(=\rho H u_{c} / \eta\right)=O(1)$ or less, where $u_{c}$ is the characteristic flow velocity. $i v$ ) The densities of fluids can be considered equal or matched; thus, the gravitational effects are neglected $[30,31,36] . v)$ The microchannel is long enough in relation to its height, i.e., $L \gg H$, to use lubrication theory [46] and neglect the end effects [47]. vi) An infinite extent of the flow domain is assumed along the $z$-axis, i.e., $W \gg H$, to disregard any effects of the sidewalls and secondary flows [46, 47]. vii) In MHD micropumps, induced magnetic fields due to the fluid motion are extremely weak and can be disregarded $[18,48]$. viii) By maintaining the electric potential across the electrodes in contact with the aqueous solution within a few $\mathrm{mV}$ around the standard equilibrium voltage of $1.23 \mathrm{~V}[8,18,49]$ and establishing an elec-

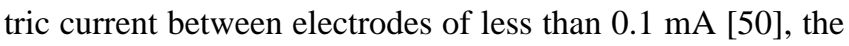
bubble formation by electrolysis can be neglected. To evaluate the orders of magnitude presented in this paragraph and throughout this work, see the physical and geometric parameters presented in Sec. 4.

With the assumptions mentioned above, a unidirectional flow and a planar liquid-liquid interface between the fluids can be considered. Specifically for the conducting fluid, the well-known Hartmann flow is recovered $[3,18,47]$. Therefore, the governing equations given by Eqs. (1) and (2) are simplified in Cartesian coordinates for the Newtonian fluid as

$$
0=-\frac{d p}{d x}+\mu \frac{d^{2} u_{1}}{d y^{2}}
$$

and for the non-Newtonian fluid as

$$
\begin{aligned}
0 & =-\frac{d p}{d x}+m \frac{d}{d y}\left[\left|\frac{d u_{2}}{d y}\right|^{n-1}\left(\frac{d u_{2}}{d y}\right)\right] \\
& -\sigma B_{y}^{2} u_{2}+\sigma B_{y} E_{z},
\end{aligned}
$$

where $u_{1}=u_{1}(y)$ and $u_{2}=u_{2}(y)$ are the fluid velocities, $E_{z}$ is the electric field on the $z$-axis, and the pressure gradient, provided by a syringe pump, is defined as $d p / d x=p_{x}=\left(p_{\text {out }}-p_{\text {in }}\right) / L$.

\subsection{Boundary conditions}

To solve the flow field established by Eqs. (6) and (7), the following boundary conditions are presented depending on the type of interface, beginning with the no-slip boundary conditions on the walls located at the upper and lower solid-liquid interfaces of the channel, that is,

$$
u_{2}=0 \quad \text { at } \quad y=H
$$

and

$$
u_{1}=0 \quad \text { at } \quad y=0 .
$$

For the liquid-liquid interface at $y=y_{1}$, the velocity continuity condition is considered as follows:

$$
u_{2}=u_{1},
$$

together with the shear stress balance condition

$$
m\left|\frac{d u_{2}}{d y}\right|^{n-1}\left(\frac{d u_{2}}{d y}\right)=\mu \frac{d u_{1}}{d y} .
$$

\subsection{Dimensionless mathematical model}

To normalize the mathematical model that describes the MHD flow behavior, the following dimensionless variables are introduced:

$$
\bar{y}=\frac{y}{H}, \quad \bar{u}_{1,2}=\frac{u_{1,2}}{u_{c}},
$$

where the characteristic velocity is defined by $u_{c}=$ $\sigma_{r e f} E_{z} B_{y} H^{2} / \eta_{\text {ref }}$ [48]; the subscript "ref" indicates a reference condition for aqueous electrolyte solutions with $T_{\text {ref }}=298.15 \mathrm{~K}$ [51-55]. Hence, the momentum conservation equations described in Eqs. (6) and (7) can be written in dimensionless form as

$$
0=-\Gamma+\bar{\mu} \frac{d^{2} \bar{u}_{1}}{d \bar{y}^{2}}
$$

and

$$
\begin{gathered}
0=-\Gamma+\bar{\eta} \frac{d}{d \bar{y}}\left[\left|\frac{d \bar{u}_{2}}{d \bar{y}}\right|^{n-1}\left(\frac{d \bar{u}_{2}}{d \bar{y}}\right)\right] \\
-H a^{2} \bar{u}_{2}+\Omega^{*} H a^{2},
\end{gathered}
$$

where the dimensionless parameters that appear are

$$
\begin{aligned}
\Gamma & =\frac{p_{x} H^{2}}{\eta_{r e f} u_{c}}, \quad \bar{\mu}=\frac{\mu}{\eta_{\text {ref }}}, \quad \bar{\eta}=\frac{\eta}{\eta_{\text {ref }}}, \\
H a & =H B_{y} \sqrt{\frac{\sigma}{\eta_{\text {ref }}}}, \quad \Omega^{*}=\frac{E_{z}}{u_{c} B_{y}},
\end{aligned}
$$

where $\Gamma$ is the ratio of pressure to magnetic forces, while $\bar{\mu}$ and $\bar{\eta}$ represent the viscosity ratios of the Newtonian and non-Newtonian fluid, respectively. As a reference for this work, consider $p_{x} \sim \sigma E_{z} B_{y}[11,48]$, whose sign depends on whether the pressure gradient favors or adversely affects the flow. Therefore, $\Gamma \sim\left( \pm \sigma / \sigma_{\text {ref }}\right)$. On the other hand, $H a$ is the Hartmann number and indicates the relationship between the magnetic forces and the viscous forces, and $\Omega^{*}$ is the load parameter. Here, $\eta \sim m\left(u_{c} / H\right)^{n-1}$ represents the order of magnitude of the non-Newtonian fluid viscosity. The parameter $\Omega^{*} H a^{2}=u_{c, 2} / u_{c}$ indicates the ratio of magnetic effects acting on a power-law fluid compared with the influence on a reference Newtonian fluid, where $u_{c, 2} \sim \sigma E_{z} B_{y} H^{2} / \eta$. Derived from the physical and geometric conditions of the microchannel system in this work, the following orders of magnitude are considered: $\Gamma=O(1), \bar{\mu}=O(1), \bar{\eta}=O(1)$, $H a \ll 1, \Omega^{*} \gg 1$ and $0 \leq \Omega^{*} H a^{2} \leq 100$.

Following the same procedure to obtain the dimensionless expressions of the boundary conditions, which consists 
of substituting Eq. (12) into Eqs. (8)-(11), the following results were achieved. For the solid-liquid interfaces, the noslip conditions on the walls of the microchannel are

$$
\bar{u}_{2}=0 \quad \text { at } \quad \bar{y}=1
$$

and

$$
\bar{u}_{1}=0 \quad \text { at } \quad \bar{y}=0 .
$$

For the liquid-liquid interface at $\bar{y}=\bar{y}_{1}$, the velocity continuity condition is

$$
\bar{u}_{2}=\bar{u}_{1},
$$

together with the shear stress balance condition

$$
\bar{\eta}\left|\frac{d \bar{u}_{2}}{d \bar{y}}\right|^{n-1}\left(\frac{d \bar{u}_{2}}{d \bar{y}}\right)=\bar{\mu} \frac{d \bar{u}_{1}}{d \bar{y}} .
$$

\section{Solution methodology}

\subsection{Velocity profile for the Newtonian fluid}

For the Newtonian fluid, the velocity profile is quickly found by integrating Eq. (13) twice with respect to the transverse coordinate $\bar{y}$, yielding

$$
\bar{u}_{1}=\frac{\Gamma}{2 \bar{\mu}} \bar{y}^{2}+C_{1} \bar{y}+C_{2},
$$

where $C_{1}$ and $C_{2}$ are integration constants.

\subsection{Velocity profile for the power-law fluid}

To obtain the velocity profile of the non-Newtonian fluid layer, first, the Hartmann number is assumed to be very small, i.e., $H a \ll 1$, due to the microchannel scale and the type of buffer solution $[18,56]$, causing the third term on the righthand side of Eq. (14), known as the Hartmann breaking term, to be neglected, yielding

$$
0=-\Gamma+\bar{\eta} \frac{d}{d \bar{y}}\left[\left|\frac{d \bar{u}_{2}}{d \bar{y}}\right|^{n-1}\left(\frac{d \bar{u}_{2}}{d \bar{y}}\right)\right]+\Omega^{*} H a^{2} .
$$

Then, integrating once with respect to the transverse coordinate $\bar{y}$, in Eq. (21) results in

$$
\left|\frac{d \bar{u}_{2}}{d \bar{y}}\right|^{n-1}\left(\frac{d \bar{u}_{2}}{d \bar{y}}\right)=\lambda\left(C_{3}-\bar{y}\right),
$$

where $C_{3}$ is an integration constant, and $\lambda=(-\Gamma+$ $\left.\Omega^{*} H a^{2}\right) / \bar{\eta}$, which may be positive or negative. On the other hand, when the velocity profile of the power-law fluid has a maximum or minimum in the range $\bar{y}_{1} \leq \bar{y} \leq 1$, the nonNewtonian fluid has two regions corresponding to positive and negative signs for the velocity gradient, being positive for region-A and negative for region-B (see Fig. 1), a circumstance that must be considered within the absolute value of Eq. (22) $[57,58]$. Equation (22) for each case is then rewritten as follows:

$$
\left(\frac{d \bar{u}_{2, A}}{d \bar{y}}\right)^{n-1}\left(\frac{d \bar{u}_{2, A}}{d \bar{y}}\right)=\lambda\left(C_{3, A}-\bar{y}\right)
$$

and

$$
\left(-\frac{d \bar{u}_{2, B}}{d \bar{y}}\right)^{n-1}\left(\frac{d \bar{u}_{2, B}}{d \bar{y}}\right)=\lambda\left(C_{3, B}-\bar{y}\right),
$$

where $C_{3, A}$ and $C_{3, B}$ are the corresponding integration constants for region-A and region- $\mathrm{B}$, respectively. Hence, Eqs. (23) and (24) can be simplified as follows:

$$
\frac{d \bar{u}_{2, A}}{d \bar{y}}=\lambda^{s}\left(C_{3, A}-\bar{y}\right)^{s} \quad \text { for region-A }
$$

and

$$
\frac{d \bar{u}_{2, B}}{d \bar{y}}=-\lambda^{s}\left(\bar{y}-C_{3, B}\right)^{s} \quad \text { for region-B, }
$$

where $s=1 / n$, and to avoid any singularity with the $\lambda$ sign in the process, $\lambda^{s}=\lambda|\lambda|^{s-1}$ [58]. Finally, the velocity profile for each section is obtained by integrating Eqs. (25) and (26), yielding the following expressions:

$$
\bar{u}_{2, A}=-\frac{\lambda|\lambda|^{s-1}}{(s+1)}\left(C_{3, A}-\bar{y}\right)^{s+1}+C_{4, A}
$$

for region-A

and

$$
\bar{u}_{2, B}=-\frac{\lambda|\lambda|^{s-1}}{(s+1)}\left(\bar{y}-C_{3, B}\right)^{s+1}+C_{4, B}
$$

for region-B,

where $C_{4, A}$ and $C_{4, B}$ are integration constants.

\subsubsection{Analysis of the fictitious interface at $\bar{y}_{2}$}

To determine the values of the integration constants $C_{3, A}$, $C_{3, B}, C_{4, A}$ and $C_{4, B}$, first, the boundary conditions corresponding to the fictitious interface position $\bar{y}=\bar{y}_{2}$ are established, which can be represented in dimensionless form as the velocity continuity condition

$$
\bar{u}_{2, B}=\bar{u}_{2, A}
$$

and the shear stress balance condition

$$
\begin{gathered}
\bar{\eta}\left(-\frac{d \bar{u}_{2, B}}{d \bar{y}}\right)^{n-1}\left(\frac{d \bar{u}_{2, B}}{d \bar{y}}\right) \\
=\bar{\eta}\left(\frac{d \bar{u}_{2, A}}{d \bar{y}}\right)^{n-1}\left(\frac{d \bar{u}_{2, A}}{d \bar{y}}\right) .
\end{gathered}
$$

However, since both sides of Eq. (30) refer to the same fluid, from Eqs. (23) and (24), the shear stress balance condition can be rewritten as follows:

$$
\lambda\left(C_{3, B}-\bar{y}\right)=\lambda\left(C_{3, A}-\bar{y}\right) .
$$


For $\bar{y}=\bar{y}_{2}$, Eq. (31) results in

$$
C_{3, B}=C_{3, A} .
$$

In addition to the aforementioned boundary conditions, we can also assume that the maximum or minimum in the velocity profile is reached at $\bar{y}=\bar{y}_{2}$, which means that the velocity gradient is equal to zero at the interface position $\bar{y}_{2}$ :

$$
\lambda^{s}\left(C_{3, A}-\bar{y}_{2}\right)^{s}=0
$$

and

$$
-\lambda^{s}\left(\bar{y}_{2}-C_{3, B}\right)^{s}=0 .
$$

In this process, Eqs. (33) and (34) were obtained from Eqs. (25) and (26), respectively. As a result of Eqs. (33) and (34), $C_{3, A}=C_{3, B}=\bar{y}_{2}$. On the other hand, applying the velocity continuity condition, i.e., substituting Eqs. (27) and (28) into Eq. (29), yields

$$
\begin{aligned}
-\frac{\lambda|\lambda|^{s-1}}{(s+1)} & \left(\bar{y}-C_{3, B}\right)^{s+1}+C_{4, B} \\
& =-\frac{\lambda|\lambda|^{s-1}}{(s+1)}\left(C_{3, A}-\bar{y}\right)^{s+1}+C_{4, A} .
\end{aligned}
$$

For $\bar{y}=\bar{y}_{2}$, with $\bar{y}_{2}=C_{3, B}=C_{3, A}$, Eq. (35) yields

$$
C_{4, B}=C_{4, A} .
$$

Therefore, $C_{3}=C_{3, B}=C_{3, A}$ and $C_{4}=C_{4, B}=C_{4, A}$. Eqs. (27) and (28) can then be rewritten as

$$
\bar{u}_{2, A}=-\frac{\lambda|\lambda|^{s-1}}{(s+1)}\left(C_{3}-\bar{y}\right)^{s+1}+C_{4} \quad \text { for region-A }
$$

and

$$
\bar{u}_{2, B}=-\frac{\lambda|\lambda|^{s-1}}{(s+1)}\left(\bar{y}-C_{3}\right)^{s+1}+C_{4} \text { for region-B. }
$$

As a result of this analysis, it is concluded that the integration constant $C_{3}$ corresponds to the position of the fictitious interface $\bar{y}_{2}$, while $C_{4}$ corresponds to the maximum or minimum velocity of the power-law fluid at that position.

\subsection{Application of boundary conditions at real inter- faces}

To find the integration constants $C_{1}, C_{2}, C_{3}$ and $C_{4}$ of the velocity profiles given in Eqs. (20), (37) and (38), the boundary conditions given by Eqs. (16)-(19) must be applied. First, Eq. (16) is substituted into Eq. (38) at $\bar{y}=1$, yielding

$$
0=-\frac{\lambda|\lambda|^{s-1}}{(s+1)}\left(1-C_{3}\right)^{s+1}+C_{4} .
$$

Second, Eq. (17) is substituted into Eq. (20) at $\bar{y}=0$, resulting in

$$
0=C_{2} \text {. }
$$

Third, Eqs. (20) and (37) are substituted into Eq. (18) at $\bar{y}=\bar{y}_{1}$, yielding the following expression:

$$
\begin{aligned}
0= & -\frac{\lambda|\lambda|^{s-1}}{(s+1)}\left(C_{3}-\bar{y}_{1}\right)^{s+1}+C_{4} \\
& -\frac{\Gamma}{2 \bar{\mu}} \bar{y}_{1}^{2}-C_{1} \bar{y}_{1}-C_{2} .
\end{aligned}
$$

Fourth, Eqs. (20) and (23) are substituted into Eq. (19) at $\bar{y}=\bar{y}_{1}$, where it is considered that $C_{3, A}=C_{3}$, yielding

$$
0=\bar{\eta}\left[\lambda\left(C_{3}-\bar{y}_{1}\right)\right]-\bar{\mu}\left(\frac{\Gamma}{\bar{\mu}} \bar{y}_{1}+C_{1}\right) .
$$

To find the constants $C_{1}, C_{3}$ and $C_{4}$, the NewtonRaphson method [59] is used to solve the system of nonlinear equations formed by Eqs. (39), (41) and (42); here, the convergence criterion is $10^{-6}$, and the proposed initial value is 1 .

\subsection{Flow rate}

The total dimensionless flow rate is determined by integrating the velocity profile of each fluid layer with respect to the transverse coordinate $\bar{y}$, which means that

$$
\bar{Q}_{T}=\bar{Q}_{1}+\bar{Q}_{2} .
$$

Here, $\bar{Q}_{1}$ represents the flow rate of the Newtonian fluid, which is defined as

$$
\bar{Q}_{1}=\int_{0}^{\bar{y}_{1}} \bar{u}_{1} d \bar{y},
$$

while $\bar{Q}_{2}$ corresponds to the flow rate of the non-Newtonian fluid, given by

$$
\bar{Q}_{2}=\int_{\bar{y}_{1}}^{\bar{y}_{2}} \bar{u}_{2, A} d \bar{y}+\int_{\bar{y}_{2}}^{1} \bar{u}_{2, B} d \bar{y} .
$$

Solving the integrals and taking into account that $C_{2}=0$ and $\bar{y}_{2}=C_{3}$ leads to

$$
\bar{Q}_{1}=\frac{\Gamma}{6 \bar{\mu}} \bar{y}_{1}^{3}+\frac{C_{1}}{2} \bar{y}_{1}^{2}
$$

and

$$
\begin{aligned}
\bar{Q}_{2} & =C_{4}\left(1-\bar{y}_{1}\right)-\frac{\lambda|\lambda|^{s-1}}{(s+1)(s+2)} \\
& \times\left[\left(1-C_{3}\right)^{s+2}+\left(C_{3}-\bar{y}_{1}\right)^{s+2}\right] .
\end{aligned}
$$

Finally, a mathematical expression for the total flow rate is found by substituting Eqs. (46) and (47) into Eq. (43), leading to

$$
\begin{aligned}
\bar{Q}_{T} & =\frac{\Gamma}{6 \bar{\mu}} \bar{y}_{1}^{3}+\frac{C_{1}}{2} \bar{y}_{1}^{2}+C_{4}\left(1-\bar{y}_{1}\right)-\frac{\lambda|\lambda|^{s-1}}{(s+1)(s+2)} \\
& \times\left[\left(1-C_{3}\right)^{s+2}+\left(C_{3}-\bar{y}_{1}\right)^{s+2}\right],
\end{aligned}
$$


where the dimensionless variable for this process is $\bar{Q}=$ $Q / Q_{c} ; Q_{c}=u_{c} H$ represents the characteristic flow rate. All aforementioned values are per unit of the channel width.

\section{Results and discussion}

For this result analysis, the following physical and geometric parameters are used to establish the corresponding values of the dimensionless parameters: $10<H \leq 500 \mu \mathrm{m}, 0 \leq \sigma \leq$ $10 \mathrm{~S} \mathrm{~m}^{-1}, 0.5<n \leq 1.5,10^{-2} \leq \mu \leq 10^{-4} \mathrm{~N} \mathrm{~s} \mathrm{~m}^{-2}$, $10^{-2} \leq m \leq 10^{-4} \mathrm{~N} \mathrm{~s}^{n} \mathrm{~m}^{-2}, E_{z} \leq 10^{5} \mathrm{~V} \mathrm{~m}^{-1}$ and $B_{y} \leq 1$ $\mathrm{T}$. The following references $[8,14,15,18,20,23,60,61]$ can be consulted to verify and deduce the ranges of values previously mentioned.

\subsection{Velocity field}

In Fig. 2, the transport of two immiscible fluids driven by purely MHD forces is analyzed. Figure 2a) presents the velocity profile as a function of the dimensionless transverse
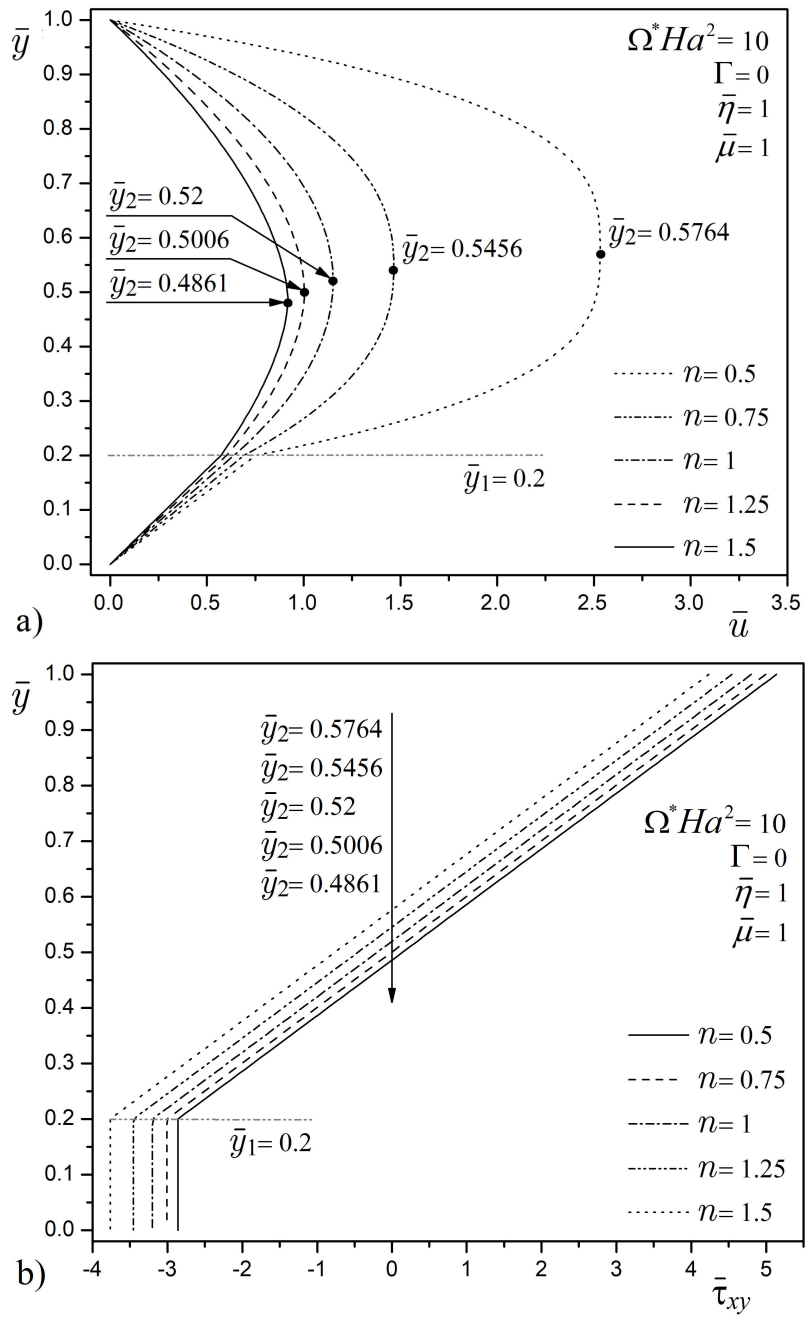

FIGURE 2. Dimensionless a) velocity profile and b) shear stress distribution for a purely MHD flow for different values of the flow behavior index $n$. coordinate $\bar{y}$, as well as the combination of the dimensionless parameters shown in the graph. The liquid-liquid interface position $\bar{y}_{1}=0.2$ indicates the spatial distribution of each fluid layer in the channel, where it can be observed that most of the space corresponds to the non-Newtonian fluid layer. Furthermore, considering that the Newtonian fluid is electrically non-conducting, the magnitude of the velocity profile depends mainly on the electrical properties of the power-law fluid, while the Newtonian fluid moves due to viscous drag effects. In this graph, the results are based on different values of the flow behavior index $n$ in the range of $0.5 \leq n \leq 1.5$. Shear-thickening fluids (also called dilatant fluids) are obtained for values of $n>1$, while shear-thinning fluids (also called pseudoplastic fluids) result for $n<1$, and when $n=1$, the case of a Newtonian fluid is recovered. Taking into account that the viscosity $\bar{\eta}$ is a function of the rate-of-strain tensor $\dot{\gamma}$ (see Eq. (5)), the highest velocity is achieved when $n=0.5$ due to the fact that for a shear-thinning fluid, an increase in the shear rate will reduce the viscosity [57], also causing fluid conduction to improve. The opposite effect occurs for a shear-thickening fluid, i.e., the lowest velocity is reached when $n=1.5$. Although the velocity increase is more pronounced in the central region of the power-law fluids, a significant difference is shown between the velocity profiles. This difference tends to diminish when approaching the liquid-liquid and solid-liquid interfaces due to the viscous drag resistance and the no-slip boundary condition, respectively. It is clear that the use of shear-thinning fluids increases the velocity of the non-conducting Newtonian fluid. On the other hand, the point $\bar{y}_{2}$ where the maximum velocity is located in the non-Newtonian fluid layer, moves away from the liquid-liquid interface position $\bar{y}_{1}$ when the value of the flow behavior index $n$ decreases. Correspondingly, in Fig. 2b), the shear stress distribution as a function of the transverse coordinate $\bar{y}$ is shown, for the same combination of dimensionless parameters as in Fig. 2a). The generalized relationship for the dimensionless shear stress for any fluid in the present investigation is obtained from Eq. (4) as

$$
\bar{\tau}_{x y}=-\bar{\eta}|d \bar{u} / d \bar{y}|^{n-1}(d \bar{u} / d \bar{y}),
$$

where $\bar{\tau}_{x y}=\tau_{x y} H / \eta_{r e f} u_{c}$. Therefore, the shear stress for the non-Newtonian fluid is determined from Eq. (49) and with the aid of Eq. (22), yielding the expression $\bar{\tau}_{x y, 2}=$ $-\bar{\eta} \lambda\left(C_{3}-\bar{y}\right)$ in the range of $\bar{y}_{1} \leq \bar{y} \leq 1$. In the case of the Newtonian fluid, the shear stress is determined from Eq. (49) together with Eq. (20), and by considering that $n=1$ and $\bar{\eta}=\bar{\mu}$, obtaining the relationship of $\bar{\tau}_{x y, 1}=-\Gamma \bar{y}-\bar{\mu} C_{1}$ applied at $0 \leq \bar{y} \leq \bar{y}_{1}$. As can be seen in Fig. 2b), the greatest shear stresses are located at the extreme limits of the driving non-Newtonian fluid, i.e., at the liquid-liquid interface placed at $\bar{y}=\bar{y}_{1}$ and the solid-liquid interface placed at $\bar{y}=1$, locations where the fluid offers the highest flow resistance. In this context, the non-Newtonian fluid offers the lowest resistance to flow when $\bar{\tau}_{x y, 2}=0$ at the positions $\bar{y}_{2}=0.5764,0.5456,0.5200,0.5006$, and 0.4861 for 
$n=0.5,0.75,1,1.25$, and 1.5 , respectively. The aforementioned finding indicates that the slope or velocity gradient at these positions is $d u_{2} / d y=0$ (see Fig. 2a) for how the velocity profile reaches the maximum velocity and the change in sign of the velocity gradient occurs for the rheological powerlaw model), which occurs when $\bar{y}_{2}=C_{3}$. In the case of the Newtonian fluid that lies between the limits $\bar{y}=0$ and $\bar{y}=\bar{y}_{1}$, both the shear stress and the velocity gradient remain constant (see Fig. 2a) for the slanted velocity profile).

Figure 3 shows an increase in the velocity profile due to an increment in the magnetic effects applied to the powerlaw fluid; compared to the influence on a reference Newtonian fluid, this behavior is reflected by the parameter $\Omega^{*} \mathrm{Ha}^{2}$, which is sensitive to the reference conditions. For this case, the flow behavior index is $n=1.4$, describing a shearthickening fluid. Furthermore, it can be seen that the mag-

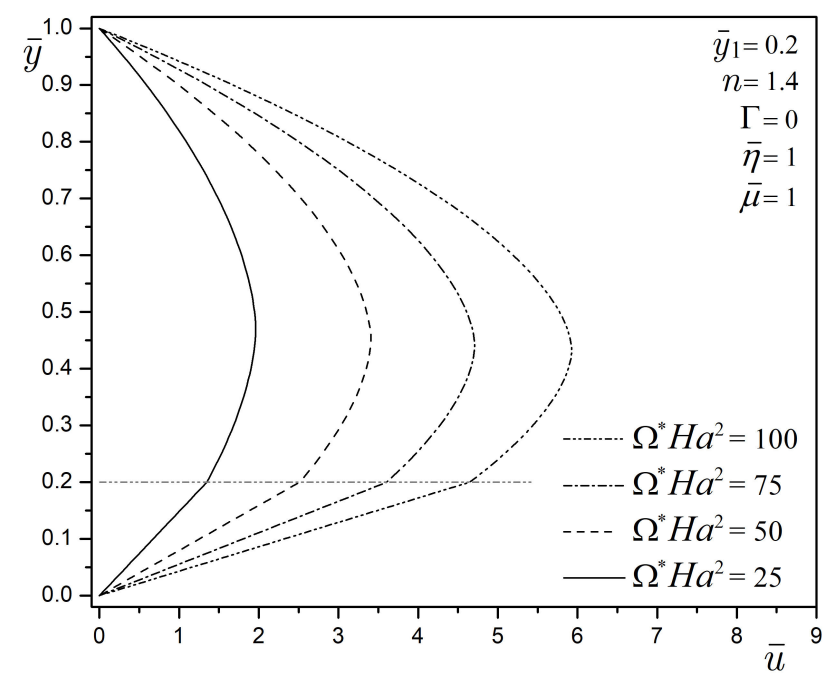

FIGURE 3. Dimensionless velocity profile for a purely MHD flow for different values of $\Omega^{*} H a^{2}$.

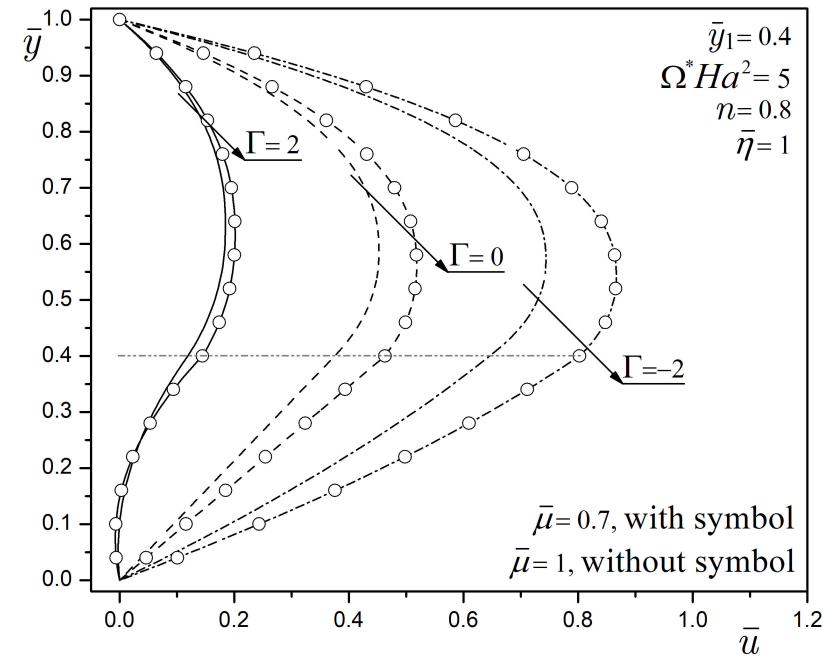

FIGURE 4. Dimensionless velocity profile for the combined MHD and pressure-driven flow for different values of $\Gamma$ and $\bar{\mu}$. netic effects act on transverse coordinate $\bar{y}$, which creates the parabolic shape in the flow field of the power-law fluid. This result is possible when the Hartmann number is assumed to be very small, i.e., $H a \ll 1$, since in this limit, the Hartmann breaking term is neglected. However, when the velocity profile reaches the liquid-liquid interface at $\bar{y}=\bar{y}_{1}$, it exhibits a linear behavior since the Newtonian fluid is not an electric conductor, and its displacement is due to the viscous drag effect. Additionally, it is shown that the velocity of the nonconducting Newtonian fluid increases significantly with the parameter $\Omega^{*} H a^{2}$ compared with the previous Fig. 2 where $\Omega^{*} H a^{2}=10$ was used.

Figure 4 analyzes the velocity field of the viscous micropump under combined MHD and pressure-driven effects; here, three values of parameter $\Gamma(=-2,0,2)$ and two values of the dynamic viscosity of the non-conducting Newtonian fluid $\bar{\mu}(=0.7,1)$ are used. The pumped fluid is a shearthinning fluid with $n=0.8$. The pressure gradient values depend on the pressure exerted at the inlet and outlet of the channel, which is generated with a syringe pump. Under this scheme, the positive values are reached when $p_{\text {out }}-p_{\text {in }}>0$ and negative values result when $p_{\text {out }}-p_{\text {in }}<0$, while for $p_{\text {out }}-p_{\text {in }}=0$ the pressure gradient effects are considered null and the purely magnetohydrodynamic flow is consequently recovered. For the first case $\Gamma=2$, the pressure at the outlet is higher than that at the channel inlet, which means that the pressure gradient is applied in the negative direction of the $x$-axis, opposing the Lorentz forces; as a result, the lowest velocity profiles in magnitude are obtained. This behavior is shown by the solid lines, where it is observed that the power-law fluid moves in the same direction as the flow, and the Newtonian fluid moves opposite to the flow because it is only affected by pressure forces. For the second case $\Gamma=0$, the only driving forces are the magnetic effects exerted by the parameter $\Omega^{*} H a^{2}=5$. For the third case $\Gamma=-2$, the pressure at the inlet is higher than that at the channel outlet, the pressure forces are in the same direction as the flow, and consequently, greater magnitude velocity profiles are achieved. The linear velocity profile of the Newtonian fluid is lost when the pressure gradient is imposed, i.e., when $\Gamma=-2$ and $\Gamma=2$. Additionally, the viscosity parameter turns out to be relevant in fluid conduction since it is responsible for regulating the resistance to movement offered by the fluid layer. For de above, by decreasing the viscosity ratio $\bar{\mu}=1$ (lines without symbols) to $\bar{\mu}=0.7$ (lines with symbols), the Newtonian fluid pumping is increased in favor of or against the flow, and the pressure gradient effects magnify this situation.

The purely MHD flow is shown in Fig. 5. As previously mentioned, shear-thinning fluids have better conduction than shear-thickening fluids. The aforementioned is reflected by the magnitude difference between lines with symbols for $n=0.6$ compared to lines without symbols for $n=1.2$. On the other hand, the flow resistance imposed by the power-law fluid is represented by the viscosity ratio $\bar{\eta}$ in the range of 0.7 to 1.3 , which means that the flow resistance is lower for 


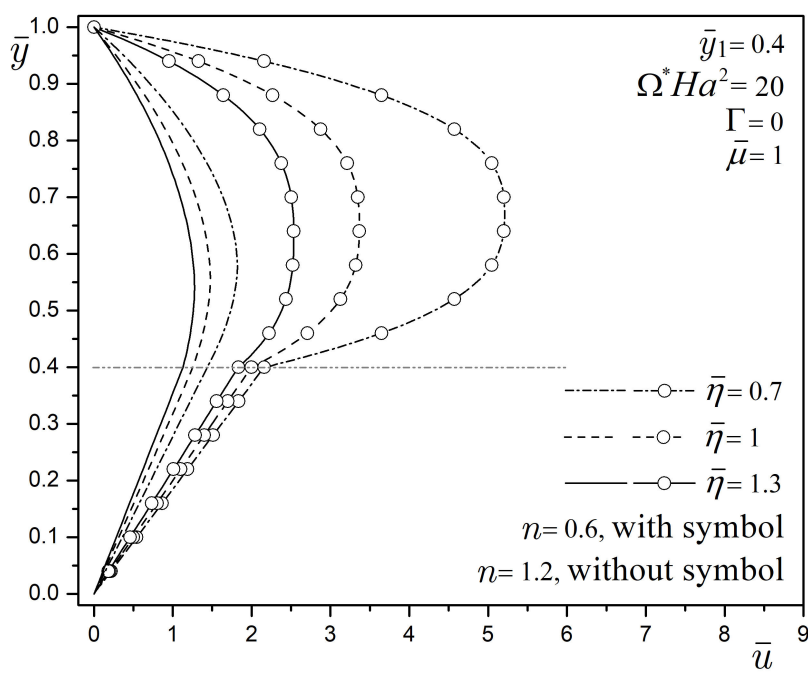

FIGURE 5. Dimensionless velocity profile for a purely MHD flow for different values of the viscosity ratio $\bar{\eta}$ and flow behavior in$\operatorname{dex} n$.

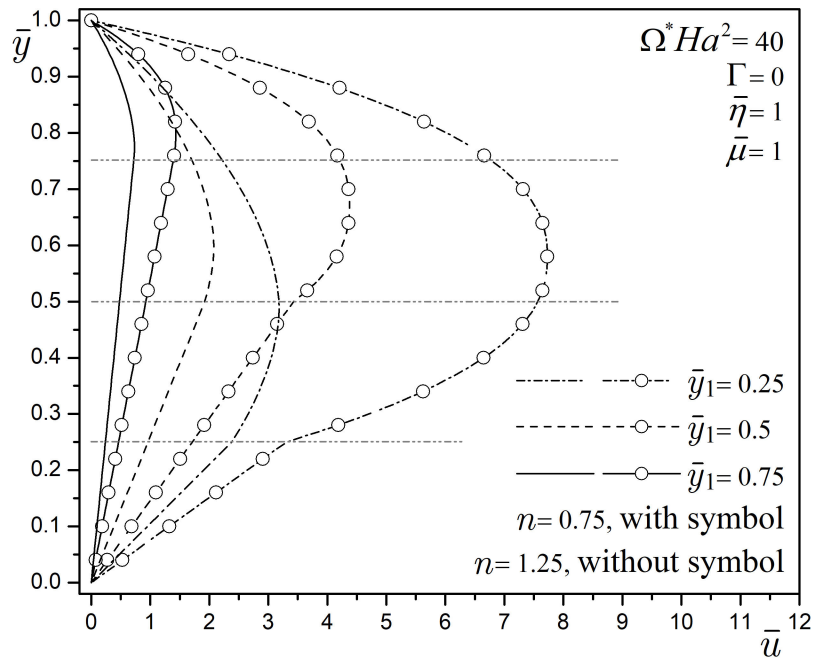

FIGURE 6. Dimensionless velocity profile for a purely MHD flow for different values of the interface position $\bar{y}_{1}$ and flow behavior index $n$.

values less than unity, which is transmitted as better conduction of the Newtonian fluid. In comparison, for values greater than unity, the flow resistance increases, and lower conduction of the Newtonian fluid is consequently presented. Finally, $\bar{\eta}=1$ indicates that the viscosity of the reference Newtonian fluid is reached. In this sense, the highest velocity magnitudes are achieved when the viscosity offers less resistance to flow, which is achieved with $\bar{\eta}=0.7$.

Figure 6 represents the micropump flow field formed by two layers of immiscible fluids, where, as in the previous figures, the power-law fluid works as a driving fluid and the Newtonian fluid as a driven fluid. Here, two cases corresponding to the driving fluid are presented, the first for a shear-thinning fluid of $n=0.75$ and the second for a shearthickening fluid of $n=1.25$. In addition to this, three liquidliquid interface positions (given by $\bar{y}_{1}=0.25,0.5,0.75$ ) that modify the thickness of each fluid layer are established. These interface positions $\bar{y}_{1}$ are selected to analyze the behavior of the flow field, taking into account the following situations when the thickness of the non-conducting fluid is less than, equal to or greater than the thickness of the conducting fluid. However, the number of possible combinations is not limited to only these three values. As can be seen, when the thickness of the driving fluid is greater than that of the driven fluid (for $\bar{y}_{1}=0.25$ ), due to the application of the Lorentz forces in a larger cross-section, velocity profiles with greater magnitude are obtained. Contrariwise, when the thickness of the driving fluid is less than that of the driven fluid (for $\bar{y}_{1}=0.75$ ), velocity profiles with less magnitude are obtained. The aforementioned situation occurs for both cases of driving fluid with $n=0.75$ and $n=1.25$.

On the other hand, we should remember that the the fictitious interface position $\bar{y}_{2}$ represents an important aspect in flow field analysis for the power-law conducting fluid since it allows us to know the location of the maximum or minimum in the velocity profile, as well as the location where the change in sign of the velocity gradient occurs. Therefore, Table I shows the interface position $\bar{y}_{2}$ of Figs. 2 and 3, while Table II displays the corresponding interface position $\bar{y}_{2}$ of Figs. 4 and 5. From Tables I and II, it is determined that all the dimensionless parameters analyzed in this work influence

TABLE I. Comparison of the interface position $\bar{y}_{2}$ under the influence of the flow behavior index $n$ and the parameter $\Omega^{*} H a^{2}$.

\begin{tabular}{cccc}
\hline \multicolumn{2}{c}{ Fig. 2 } & \multicolumn{2}{c}{ Fig. 3 } \\
Parameter & Interface & Parameter & Interface \\
$n$ & $\bar{y}_{2}$ & $\Omega^{*} \mathrm{Ha}^{2}$ & $\bar{y}_{2}$ \\
\hline 0.5 & 0.5764 & 100 & 0.4323 \\
0.75 & 0.5456 & 75 & 0.4403 \\
1 & 0.52 & 50 & 0.4513 \\
1.25 & 0.5006 & 25 & 0.4694 \\
1.5 & 0.4861 & & \\
\hline
\end{tabular}

TABLE II. Comparison of the interface position $\bar{y}_{2}$ under the influence of the pressure gradient $\Gamma$ and the viscosity ratio $\bar{\mu}$, as well as the viscosity ratio $\bar{\eta}$ and the flow behavior index $n$.

\begin{tabular}{cccc}
\hline \multicolumn{2}{c}{ Fig. 4 } & \multicolumn{2}{c}{ Fig. 5 } \\
\hline Parameter & Interface $\bar{y}_{2}$, & Parameter & Interface $\bar{y}_{2}$, \\
\hline$\Gamma$ & with $\bar{\mu}=0.7$ & $\bar{\eta}$ & with $n=0.6$ \\
\hline-2 & 0.5435 & 0.7 & 0.6699 \\
0 & 0.5620 & 1 & 0.6495 \\
2 & 0.6177 & 1.3 & 0.6284 \\
\hline$\Gamma$ & with $\bar{\mu}=1$ & $\bar{\eta}$ & with $n=1.2$ \\
\hline-2 & 0.5734 & 0.7 & 0.5710 \\
0 & 0.5877 & 1 & 0.5470 \\
2 & 0.6323 & 1.3 & 0.5301 \\
\hline
\end{tabular}




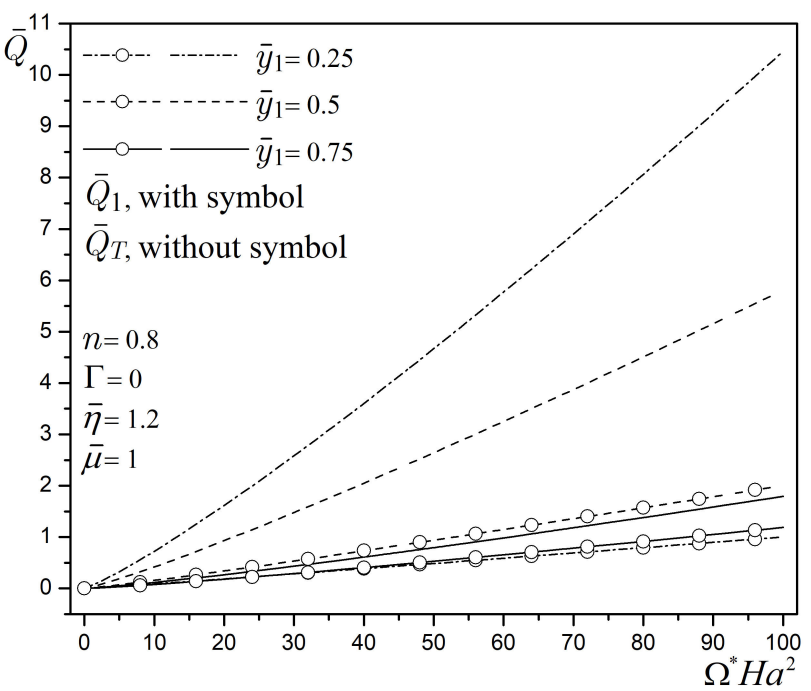

FIGURE 7. Dimensionless flow rate for a purely MHD flow as a function of the parameter $\Omega^{*} \mathrm{Ha}^{2}$ for different values of the interface position $\bar{y}_{1}$.

the position of $\bar{y}_{2}$; however, the most representative for the location of this fictitious interface is the flow behavior index.

\subsection{Flow rate}

In Fig. 7, the dimensionless flow rate $\bar{Q}$ of a purely MHD flow is analyzed as a function of the parameter $\Omega^{*} H a^{2}$, as well as for different positions of the liquid-liquid interface $\bar{y}_{1}$. Here, $\bar{Q}_{T}$ refers to the total flow rate handled by the micropump, while $\bar{Q}_{1}$ is the flow rate of the non-conducting fluid (Newtonian fluid). The driving fluid is a shear-thinning fluid with $n=0.8$ and a viscosity ratio of $\bar{\eta}=1.2$. As can be seen, the flow rate tends to increase with the increment of the parameter $\Omega^{*} H a^{2}$, reaching a maximum value when $\Omega^{*} H a^{2}=100$ and a minimum value when $\Omega^{*} H a^{2}=0$. In the absence of magnetic effects, i.e., at the hydrodynamic limit when $\Omega^{*} H a^{2} \rightarrow 0$, the classical Poiseuille flow can be recovered if $\Gamma \neq 0$ (a case that was not included in the flow rate analysis because these effects do not correspond to the main focus, which is the transport of a non-conducting fluid by viscous drag effects from a neighboring conducting fluid induced by Lorentz forces). On the other hand, the magnitude of the total flow rate $\bar{Q}_{T}$ increases when the thickness of the driving fluid increases. However, this behavior is not repeated in the case of $\bar{Q}_{1}$ since the maximum flow rate is obtained when $\bar{y}_{1}=0.5$ (which occurs when the thickness of both layers is the same). The above means that $\bar{Q}_{1}$ decreases when the liquid-liquid interface position $\bar{y}_{1}$ moves away from the microchannel center. The latter effect results because when the thickness of the Newtonian fluid layer is greater (when $\bar{y}_{1}=0.75$ ), the driving fluid is not able to pump large amounts of liquid, and when the thickness of the Newtonian fluid layer is less (when $\bar{y}_{1}=0.25$ ), the amount of pumped fluid decreases due to the reduction in cross-sectional area.

Figure 8 shows the dimensionless flow rate $\bar{Q}$ of a purely MHD flow as a function of the interface position $\bar{y}_{1}$ for dif-

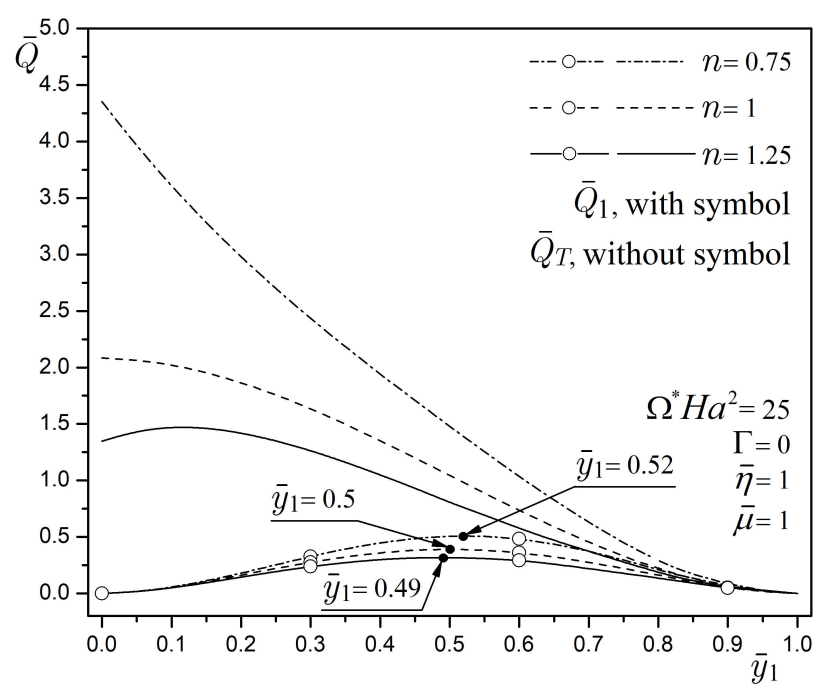

FIGURE 8.Dimensionless flow rate for a purely MHD flow as a function of the interface position $\bar{y}_{1}$ for different values of the flow behavior index $n$.

ferent values of the flow behavior index $n$. In the graph, the maximum total flow rates $\bar{Q}_{T}$ (lines without symbols) are achieved when the thickness of the driving fluid (power-law fluid) is greater than that of the driven fluid (Newtonian fluid), which happens when $\bar{y}_{1} \rightarrow 0$. However, in this condition, the driven fluid (Newtonian fluid) tends to have the minimum flow rate $\bar{Q}_{1}$ (lines with symbols) because it occupies a tiny channel cross-section until reaching the condition of $\bar{Q}_{1}=0$ at $\bar{y}_{1}=0$, which corresponds to the absence of the driven fluid. In the contrary case, $\bar{Q}_{T}$ and $\bar{Q}_{1}$ tend to diminish as $\bar{y}_{1} \rightarrow 1$ because the driving fluid is thinner, therefore diminishing the driving force to move the fluids until reaching the condition of $\bar{Q}_{T}=0$ and $\bar{Q}_{1}=0$ at $\bar{y}_{1}=1$, i.e., the absence of the driving fluid. Because the purpose of this work is to pump the non-conducting fluid (Newtonian fluid), the maximum flow $\bar{Q}_{1}$ will be obtained when the position $\bar{y}_{1}$ is located near the microchannel center, more specifically, $\bar{y}_{1}=0.49$, $\bar{y}_{1}=0.5$ and $\bar{y}_{1}=0.52$ for $n=1.25, n=1$ and $n=0.75$, respectively. Furthermore, it is clear that the flow rate $\bar{Q}_{1}$ increases with shear-thinning fluids.

Figure 9 evaluates the dimensionless flow rate as a function of the flow behavior index, which ranges from a shearthinning fluid of $n=0.5$ to a shear-thickening fluid of $n=1.5$; additionally, the analysis is performed under three flow conditions determined by the viscosity ratios of $\bar{\eta}=$ $0.6,1,1.4$. In this case, it is assumed that the thickness of the driving fluid layer is less than that of the driven fluid layer through a liquid-liquid interface located at $\bar{y}_{1}=0.6$ and that in the absence of a pressure gradient, the flow field is pumped only by magnetic effects given by $\Omega^{*} H a^{2}=25$. The results are prepared for both the total flow rate $\bar{Q}_{T}$ and the flow rate of the driven fluid $\bar{Q}_{1}$. The graph shows that the largest flow rate values correspond to shear-thinning fluids $(n<1)$ up to a maximum value of $n=0.5$, which then decrease when approaching the value of the shear-thickening fluid of $n=1.5$. 


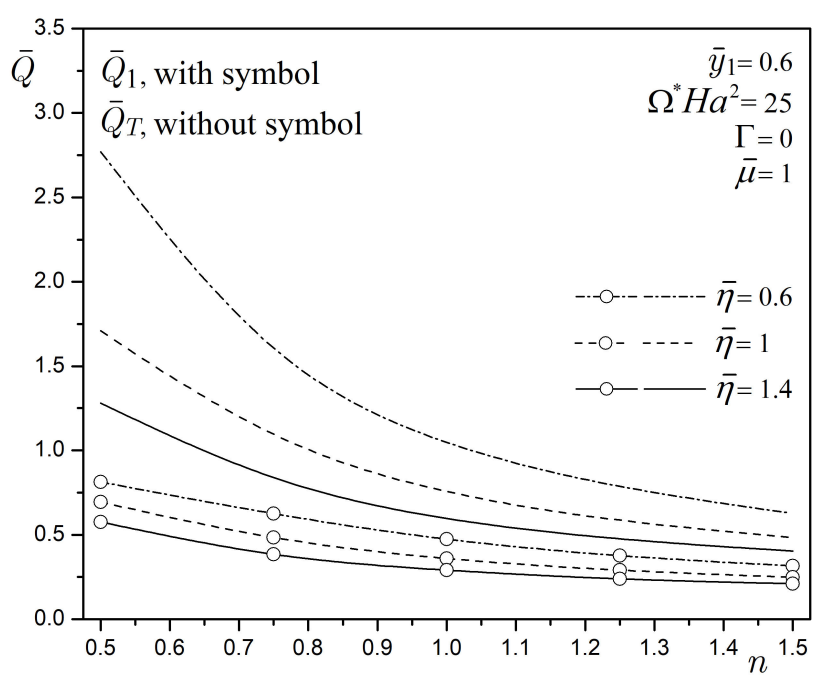

FIGURE 9. Dimensionless flow rate for a purely MHD flow as a function of the flow behavior index $n$, for different values of the viscosity ratio $\bar{\eta}$.

The changes in the viscosity ratio parameter from $\bar{\eta}=1.4$ to $\bar{\eta}=0.6$ allow for decreased resistance of the driving fluid opposed to the flow, and consequently, higher volumetric flows are achieved. This increase is shown for $\bar{Q}_{T}$ and $\bar{Q}_{1}$, indicating that in both cases, the maximum flow rate is achieved with $\bar{\eta}=0.6$ and the minimum is achieved with $\bar{\eta}=1.4$.

\section{Conclusions}

The present work studied the transport of a non-conducting fluid by viscous drag forces from a neighboring conducting fluid. The micropump pumping mechanism is based on the MHD principle, where the pumping source arises from the Lorentz forces. Some important findings that improve the flow rate of the non-conducting fluid are that the thickness values of both fluid layers (conducting and non-conducting) should be similar, i.e., the liquid-liquid interface should be located near the center of the microchannel; additionally, the flow rate is enhanced when the conducting fluid is of the shear-thinning variety. On the other hand, external pressure gradient effects and moving parts can be omitted because the magnetic Lorentz forces can provide adequate pumping of the non-conducting fluid. This study's development can contribute to understanding non-conducting immiscible fluid transport in microfluidic system applications.

\section{Acknowledgments}

This work was supported by the Instituto Politécnico Nacional in Mexico (grant numbers SIP-20210897 and SIP20211535). The authors thank the referee for his useful suggestions.
1. U. Müller and L. Bühler, Magnetofluiddynamics in Channels and Containers (Springer, Berlin-Heidelberg, 2001), https: //doi.org/10.1007/978-3-662-04405-6

2. P.A. Davidson, An Introduction to Magnetohydrodynamics (Cambridge Univerity Press, New York, U.S., 2001). https: //doi.org/10.1017/CB09780511626333.

3. R. J. Moreau, Magnetohydrodynamics (Springer, Dordrecht, 1990), https://doi.org/10.1007/ 978-94-015-7883-7

4. O.M. Al-Habahbeh, M. Al-Saqqa, M. Safi and T. Abo Khater, Review of magnetohydrodynamic pump applications, Alex. Eng. J. 55 (2016) 1347, http://doi.org/10.1016/j. aej.2016.03.001

5. V. Patel and S.K. Kassegne, Electroosmosis and thermal effects in magnetohydrodynamic (MHD) micropumps using 3D MHD equations, Sens. Actuator B-Chem. 122 (2007) 42, https: //doi.org/10.1016/j.snb.2006.05.015

6. I. Dolezel, V. Kotlan, B. Ulrych and V. Valenta, Magnetohydrodynamic pumps with permanent magnets for pumping molten metals or salts, Electroscope 2009 (2009) 86.

7. I. Dolezel, V. Kotlan and B. Ulrych, Magnetohydrodynamic pumps for molten salts in cooling loops of high-temperature nuclear reactors, Prz. Elektrotech. 2011 (2011) 28.

8. H. Kabbani, A. Wang, X. Luo, and S. Qian, Modeling Redoxbased magnetohydrodynamics in three-dimensional microfluidic channels, Phys. Fluids 19 (2007) 083604, https:// doi.org/10.1063/1.2759532
9. C. Kleinstreuer, Microfluidics and Nanofluidics. Theory and Selected Applications (Wiley, New Jersey, 2013), https: //doi.org/10.1002/9781118749890.

10. D. Li, Encyclopedia of Microfluidics and Nanofluidics (Springer, Boston, 2008). https://doi.org/10.1007/ 978-0-387-48998-8

11. A. Homsy et al., A high current density DC magnetohydrodynamic (MHD) micropump, Lab Chip 5 (2005) 466, https: //doi.org/10.1039/B417892K

12. H. S. Kabbani, M. J. Mack, S. W. Joo and S. Qian, Analytical prediction of flow field in magnetohydrodynamic-based microfluidic devices, J. Fluids Eng.-Trans. ASME 130 (2008) 091204, https://doi.org/10.1115/1.2953302

13. F. E. H. Tay, Microfluidics and BioMEMS Applications (Springer, Boston, 2002). https://doi.org/10.1007/ 978-1-4757-3534-5

14. S. Lim and B. Choi, A study on the MHD (magnetohydrodynamic) micropump with side-walled electrodes, J. Mech. Sci. Technol. 23 (2009) 739, https://doi.org/10.1007/ s12206-008-1107-0

15. L. Huang, W. Wang, M.C. Murphy, K. Lian and Z.-G. Ling, LIGA fabrication and test of a DC type magnetohydrodynamic (MHD) micropump, Microsyst. Technol. 6 (2000) 235, https://doi.org/10.1007/s005420000068. 
16. D. Chatterjee and S. Amiroudine, Lattice Boltzmann simulation of thermofluidic transport phenomena in a DC magnetohydrodynamic (MHD) micropump, Biomed. Microdevices 13 (2011) 147, https://doi.org/10.1007/ s10544-010-9480-8

17. H. H. Bau, J. Zhu, S. Qian and Y. Xiang, A magnetohydrodynamically controlled fluidic network, Sens. Actuator B Chem. 88 (2003) 205, https://doi.org/10.1016/ S0925-4005(02)00325-8

18. M. Rivero and S. Cuevas, Analysis of the slip condition in magnetohydrodynamic (MHD) micropumps, Sens. Actuator B Chem. 166-167 (2012) 884, https://doi.org/10. $1016 / j . s n b .2012 .02 .050$.

19. J. Azimi-Boulali, M. Zakeri and M. Shoaran, A study on the 3D fluid flow of MHD micropump, J. Braz. Soc. Mech. Sci. Eng. 41 (2019) 478, https://doi.org/10.1007/ s40430-019-1979-1.

20. S. Moghaddam, Investigating flow in MHD micropumps, $S N$ Appl. Sci. 1 (2019) 1609, https://doi.org/10.1007/ s42452-019-1644-4.

21. Y. Jian and L. Chang, Electromagnetohydrodynamic (EMHD) micropumps under a spatially non-uniform magnetic field, $A I P$ Adv. 5 (2015) 057121, https://doi.org/10.1063/1. 4921085

22. P. K. Mondal and S. Wongwises, Magneto-hydrodynamic (MHD) micropump of nanofluids in a rotating microchannel under electrical double-layer effect, Proc. Inst. Mech. Eng. E 234 (2020) 318, https://doi.org/10.1177/ 0954408920921697.

23. S. Moghaddam, MHD micropumping of power-law fluids: A numerical solution, Korea Aust. Rheol. J. 25 (2013) 29, https://doi.org/10.1007/s13367-013-0004-y.

24. M. Pourjafar, F. Malmir, S. Bazargan and K. Sadeghy, Magnetohydrodynamic flow of Bingham fluids in a plane channel: A theoretical study, J. Non-Newton. Fluid Mech. 264 (2019) 1, https://doi.org/10.1016/j.jnnfm.2018.12. 005.

25. Y. A. Elmaboud and S. I. Abdelsalam, DC/AC magnetohydrodynamic-micropump of a generalized Burger's fluid in an annulus, Phys. Scr. 94 (2019) 115209, https://doi.org/10.1088/1402-4896/ab206d

26. A. Shahidian et al., Flow analysis of non-Newtonian blood in a magnetohydrodynamic pump, IEEE Trans. Magn. 45 (2009) 2667, https://doi.org/10.1109/TMAG. 2009.2018954

27. F.-Q. Li, Y.-J. Jian, Z.-Y. Xie, and L. Wang, Electromagnetohydrodynamic flow of Powell-Eyring fluids in a narrow confinement, J. Mech. 33 (2017) 225, https : / / doi .org/10. $1017 /$ jmech.2016.75

28. R. Shail, On laminar two-phase flows in magnetohydrodynamics, Int. J. Eng. Sci. 11 (1973) 1103, https://doi.org/ 10.1016/0020-7225(73)90111-0

29. J. Lohrasbi and V. Sahai, Magnetohydrodynamic heat transfer in two-phase flow between parallel plates, Appl. Sci. Res. 45 (1988) 53, https://doi.org/10.1007/BF00384182
30. J. C. Umavathi, A. Mateen, A.J. Chamka and A. Al-Mudhaf, Oscillatory Hartmann two-fluid flow and heat transfer in a horizontal channel, Int. J. Appl. Mech. Eng. 11 (2006) 155.

31. A. Mateen, Magnetohydrodynamic flow and heat transfer of two immiscible fluids through a horizontal channel, Int. J. Curr. Eng. Technol. 3 (2013) 1952.

32. Z. Abbas and J. Hasnain, Two-phase magnetoconvection flow of magnetite $\left(\mathrm{Fe}_{3} \mathrm{O}_{4}\right)$ nanoparticles in a horizontal composite porous annulus, Res. Phys. 7 (2017) 574, https://doi. org/10.1016/j.rinp.2016.12.022

33. Z. Abbas, J. Hasnain and M. Sajid, Hydromagnetic mixed convective two-phase flow of couple stress and viscous fluids in an inclined channel, Z. Naturforsch. A 69 (2014) 553, https://doi.org/10.5560/zna.2014-0048

34. M. S. Malashetty and J. C. Umavathi, Two-phase magnetohydrodynamic flow and heat transfer in an inclined channel, Int. J. Multiph. Flow 23 (1997) 545, https://doi.org/10. 1016/S0301-9322(96)00068-7.

35. M. S. Malashetty, J. C. Umavathi, and J. P. Kumar, Magnetoconvection of two-immiscible fluids in vertical enclosure, Heat Mass Transf. 42 (2006) 977, https : / / doi .org/10. 1007/s00231-005-0062-x.

36. D. Nikodijevic, D. Milenkovic and Z. Stamenkovic, MHD Couette two-fluid flow and heat transfer in presence of uniform inclined magnetic field, Heat Mass Transf. 47 (2011) 1525 , https://doi.org/10.1007/s00231-011-0815-7.

37. P. Chanturani and S.S. Bharatiya, Two layered magnetohydrodynamic flow through parallel plates with applications, Indian J. Pure Appl. Math. 32 (2001) 55.

38. A. Brask, G. Goranović, M.J. Jensen and H. Bruus, A novel electro-osmotic pump design for nonconducting liquids: theoretical analysis of flow rate-pressure characteristics and stability, J. Micromech. Microeng. 15 (2005) 883, https: / / doi. org/10.1088/0960-1317/15/4/029.

39. Y. Gao, T. N. Wong, C. Yang and K. T. Ooi, Transient twoliquid electroosmotic flow with electric charges at the interface, ColloidS Surf. A. 266 (2005) 117, https : // doi.org/10. $1016 / j . c o l s u r f a .2005 .05 .068$

40. G. D. Ngoma and F. Erchiqui, Pressure gradient and electroosmotic effects on two immiscible fluids in a microchannel between two parallel plates, J. Micromech. Microeng. 16 (2006) 83, https://doi.org/10.1088/0960-1317/16/1/ 012

41. M. Liu and J. Yang, Electrokinetic effect of the endothelial glycocalyx layer on two-phase blood flow in small blood vessels, Microvas. Res. 78 (2009) 14, https : //doi.org/10. $1016 / j . m v r .2009 .04 .002$

42. A. M. Afonso, M. A. Alves and F. T. Pinho, Analytical solution of two-fluid electro-osmotic flows of viscoelastic fluids, $J$. Colloid Interface Sci. 395 (2013) 277, https : / / doi.org/ $10.1016 / j \cdot j c i s .2012 .12 .013$

43. Y. Huang, H. Li and T.N. Wong, Two immiscible layers of electro-osmotic driven flow with a layer of conducting non-Newtonian fluid, Int. J. Heat Mass Transf. 74 (2014) 368, https://doi.org/10.1016/j. ijheatmasstransfer.2014.02.068 
44. K. M. Joshep, A. Peter, P. E. Asie and S. Usman, The unsteady MHD free convective two immiscible fluid flows in a horizontal channel with heat and mass transfer, Int. J. Math. Comput. Res. 3 (2015) 954.

45. D. Nikodijević, Ž. Stamenković, D. Milenković, B. Blagojević and J. Nikodijević, Flow and heat transfer of two immiscible fluids the presence of uniform inclined magnetic field, Math. Probl. Eng. 2011 (2011) 132302, https://doi.org/10. $1155 / 2011 / 132302$

46. A. Z. Szeri, Fluid Film Lubrication 2nd ed. (Cambridge University Press, Cambridge, 2010), https://doi.org/10. $1017 / \mathrm{CBO} 9780511782022$

47. G. W. Sutton and A. Sherman, Engeneering Magnetohydrodynamics (Dover Publications, New York, 2006).

48. A. Ramos, Electrohydrodynamic and magnetohydrodynamic micro- pumps, in: S. Hardt, F. Schönfeld (Eds.), Microfluidic Technologies for Miniaturized Analysis Systems (Springer, Boston, 2007). https://doi.org/10.1007/ 978-0-387-68424-6_2

49. H. Kim, H. Hwang, S. Baek and D. Kim, Design, fabrication and performance evaluation of a printed-circuit-board microfluidic electrolytic pump for lab-on-a-chip devices, $A$. Phys. 277 (2018) 73, https://doi.org/10.1016/j. sna.2018.04.042

50. A. Homsy, Ph.D. thesis, University of Neuchâtel, 2006.

51. H.-L. Zhang and S. J. Han, Viscosity and density of water + sodium chloride + potassium chloride solutions at $298.15 \mathrm{~K}, J$. Chem. Eng. Data 41 (1996) 516, https://doi.org/10. $1021 /$ je9501402

52. T. Isono, Density, viscosity, and electrolytic conductivity of concentrated aqueous electrolyte solutions at several temperatures. Alkaline-earth chlorides, lanthanum chloride, sodium chloride, sodium nitrate, sodium bromide, potassium nitrate, potassium bromide, and cadmium nitrate, J. Chem. Eng. Data 29 (1984) 45, https://doi.org/10.1021/ je00035a016
53. J. Kestin, H. Ezzat Khalifa and R. J. Correia, Tables of the dynamic and kinematic viscosity of aqueous $\mathrm{KCI}$ solutions in the temperature range $25-150{ }^{\circ} \mathrm{C}$ and the pressure range 0.1 $35 \mathrm{MPa}$, J. Phys. Chem. Ref. Data 10 (1981) 57, https: //doi.org/10.1063/1.555640

54. Y. C. Wu, W. F. Koch and K. W. Pratt, Proposed new electrolytic conductivity primary standars for $\mathrm{KCl}$ solutions, J. Res. Natl. Inst. Stand. Technol. 96 (1991) 191, https://doi. org/10.6028/jres.096.008.

55. H. Golnabi, M.R. Matloob, M. Bahar and M. Sharifian, Investigation of electrical conductivity of different water liquids and electrolyte solutions, Iran. Phys. J. 3 (2009) 24, https://www.sid.ir/en/journal/ ViewPaper.aspx?id=191551.

56. S. Qian and H. H. Bau, Magneto-hydrodynamics based microfluidics, Mech. Res. Commun. 36 (2009) 10, https:// doi.org/10.1016/j.mechrescom.2008.06.013

57. F. A. Morrison, Undestanding Rheology (Oxford University Press, Oxford, 2001).

58. R. W. Flumerfelt, W. M. Pierick, S. L. Cooper and R. B. Bird, Generalized plane Couette flow of a non-Newtonian fluid, Ind. Eng. Chem. Fundamen. 8 (1969) 354, https://doi .org/ $10.1021 / i 160030 a 028$

59. J.D. Hoffman, Numerical Methods for Engineers and Scientists (Marcel Dekker, New York 2001).

60. J. Jang and S. S. Lee, Theoretical and experimental study of MHD (magnetohydrodynamic) micropump, Sens. Actuator A-Phys. 80 (2000) 84, https: //doi.org/10.1016/ S0924-4247(99)00302-7

61. Z. P. Aguilar, P. Arumugam and I. Fritsch, Study of magnetohydrodynamic driven flow through LTCC channel with self-contained electrodes, J. Electroanal. Chem. 591 (2006) 201, https://doi.org/10.1016/j.jelechem. 2006.04 .019 